Ebisu Ebisu

Études japonaises Études japonaises

$55 \mid 2018$

La fabrique des villes japonaises contemporaines

\title{
Grands ensembles de Tokyo : le logement public à l'épreuve des ans
}

東京団地：時の流机に立ち向加う公共住宅

Tokyo Housing Projects: Public Housing in the Face of Time

\section{Nicolas Pinet}

\section{OpenEdition}

\section{Journals}

Édition électronique

URL : http://journals.openedition.org/ebisu/2416

DOI : 10.4000/ebisu.2416

ISSN : 2189-1893

Éditeur :

Institut français de recherche sur le Japon (UMIFRE 19 MAEE-CNRS), Maison franco-japonaise

Édition imprimée

Date de publication : 15 décembre 2018

Pagination : $59-84$

ISSN : 1340-3656

\section{Référence électronique}

Nicolas Pinet, "Grands ensembles de Tokyo : le logement public à l'épreuve des ans », Ebisu [En ligne], 55 | 2018, mis en ligne le 15 décembre 2018, consulté le 01 mai 2019. URL : http:// journals.openedition.org/ebisu/2416; DOI : 10.4000/ebisu.2416 


\section{Grands ensembles de Tokyo : le logement public à l'épreuve des ans Nicolas PINET}

東京団地：時の流れに立ち向かう公共住宅

ニコラ・ピネ

Tokyo Housing Projects: Public Housing in the Face of Time

Nicolas Pinet

Mots-clés : Grands ensembles, logement public, vieillissement, villes, Japon, politiques de logement, démographie.

L'auteur: Nicolas Pinet est chercheur au Laboratoire du changement social et politique (université Paris Diderot) et chargé de cours aux universités Paris Diderot et Évry. Il conduit depuis 2011 des recherches sur les quartiers populaires et les ensembles d'habitat social.

Résumé : Les grands ensembles de logements publics ne représentent qu'un peu moins de $8 \%$ des logements de la métropole tokyoïte (2013). Cependant, par l'ampleur des projets d'aménage- ment qui leur ont donné naissance, leur extension spatiale et leur pérennité, ils constituent des marqueurs structurels du tissu urbain. Si les permanences permanence du bâti, à quelques exceptions près, et permanence des habitants, miroir grossissant du vieillissement de la population -, semblent dominer, elles induisent aussi par leur poids même une série de changements, tant au niveau des politiques de logement quau niveau des manières d'habiter. Ce texte se propose de revenir sur cette dynamique duale en s'appuyant de manière conjointe sur les données chiffrées disponibles et sur une ethnographie conduite pendant quatre ans dans un grand ensemble du nord-est de Tokyo. 
フキーワート

団地、公営住宅、高齢化、都市、日本、住宅政策、 人口学

\section{著者}

ニコラ・ピネは社会科学高等研究院で社会学 博士号を取得。パリ・ディドロ大学の社会政 治変革研究所に所属。パリ・ディドロ大学と エヴリー大学で非常勤講師を務める。2011 年から公営住宅と下町に関する研究に従事。

\section{要旨}

2013 年の統計によると東京における公共団地 の数は全住宅件数の 8 パーセントにも満たな い。しかし公共団地は都市整備計画としての重 要性、及び建築面積の広さと存続性のため、都 市のランドマークとなっている。一見すると、 その建物とそこに住む居住者が変わらないとい う不変性が公共団地の特徵ではあるが、その不 変性こそが公共住宅政策とそこでの住み方を変 化させてきた。本稿はこの事実を統計資料の分 析と東京北東部の都営住宅での四年間に及ぶ民 族誌調査をもとに明らかにする。
\ Keywords: Housing projects, public housing, ageing, cities, Japan, housing policies, demographics

The Author: Nicolas Pinet is a researcher at the Research Center on Social and Political Change (LCSP), Université Paris Diderot, and an assistant lecturer at Université Paris Diderot and Université d'Évry. Since 2011 he has carried out research on public housing and working-class Tokyo neighborhoods.

Abstract: Public housing projects account for just under $8 \%$ of Tokyo's total hou- sing (2013). However, the scale of the correlative urban development plans and the projects' extension and durability make them city landmarks. At first sight, permanency - of the buildings and their residents - seems to be their most striking characteristic. But permanency leads by its own weight to a series of changes affecting both housing policies and dwelling habits. This article explores this twofold process through an analysis of available statistical data combined with findings from a fouryear ethnography conducted in a Tokyo public housing project. 


\title{
Grands ensembles de Tokyo : le logement public à l'épreuve des ans
}

\author{
Nicolas PINET*
}

Le promeneur qui déambule un peu longuement dans Tokyo a de fortes chances de se trouver, à un moment de son parcours, face à un ou plusieurs immeubles de couleur gris-beige, plutôt anciens, souvent de cinq ou douze étages ${ }^{1}$. De même, sur les plans de la ville, des ensembles plus ou moins vastes de bâtiments alignés occupent un ou plusieurs blocs, avec des appellations associant toujours un toponyme à des syntagmes qui renvoient au type de logement et varient en fonction des endroits et des cartes : toei jūtaku 都営住宅 (logements du gouvernement métropolitain), toei apāto 都営アパート (appartements du gouvernement métropolitain), danchi 団地 (ensemble d'habitat collectif), ou parfois toei danchi 都営団地 (ensemble d'habitat collectif du gouvernement métropolitain). Dans certains cas, surtout en périphérie, leur extension est telle qu'ils constituent une véritable ville dans la ville, très visible sur les photos aériennes : c'est ainsi le cas d'Akabanedai danchi 赤羽台団地 (1962, 3373 appartements, Kita-ku), de Toshima go chōme danchi 豊島五丁目団地 (1972, 4959 appartements, Kita-ku), de Toei Kirigaoka apāto 都営桐ケ丘アパート (1959-1975, 5020

1. Les étages sont comptés comme les Japonais les comptent, le premier étage japonais correspondant au rez-de-chaussée français.

* Chercheur au Laboratoire de changement social et politique (Paris Diderot). 
appartements, Kita-ku) ou de Takashimadaira danchi 高島平団地 (1972, 10170 appartements, Itabashi-ku). Comme les dates de construction l'indiquent, ces grands ensembles ont souvent plus de $40 \mathrm{ans}^{2}$, alors que la durée de vie moyenne des logements au Japon est de 30 ans, contre 55 aux États-Unis et 77 en Angleterre (Kokudo kōtsu shō 2009 : 12). Ils constituent ainsi, par leur extension spatiale et leur longévité, des marqueurs structurels du tissu urbain tokyoïte. Ils occupent aussi une place particulière dans l'imaginaire culturel et mémoriel, tout spécialement avec l'engouement récent pour les années 30 de l'ère Shōwa (1955-1964). En témoignent la multiplication des publications depuis une dizaine d'années (Ishimoto 2008; Ōyama 2008; Shiki 2012; Haseda 2015; Tökyōjin 371 gō tokushū "Tōkyō natsukashi no danchi» 2016), ou les films ${ }^{3}$, romans (Shigematsu 2015) et mangas (Oda 2004; Ms 2011a, 2011b; Imai 2011a, 2011b) qui les ont mis en scène.

La diversité des noms donnés à ces grands ensembles révèle que, derrière leur apparence similaire - architecture, vétusté souvent -, les types de logements offerts ne sont pas identiques. Un observateur français aura tendance à y voir la déclinaison japonaise des grands ensembles d'habitat social construits en France à partir des années 1950. Mais en fait, seule une partie de ces bâtiments a une vocation sociale. Saisir le panorama actuel des ensembles d'habitat collectif suppose donc de se doter d'abord de clés de lecture. Comme souvent, le présent peut être lu à l'aune du passé et la situation des grands ensembles à l'aune des politiques de logement mises en place à partir des années 1950. Cette mise en perspective préalable

2. Selon les chiffres officiels, $33,7 \%$ des logements publics ont été construits avant 1974 (44 ans en 2018), 26,8 \% entre 1975 et 1984 (plus de 34 ans), 18,8\% entre 1985 et 1994 (plus de 24 ans) et 20,7 \% entre 1995 et 2012 (Housing Policy Division, Housing Bureau, Ministry of Land, Infrastructure, Transport and Tourism 2016 : 28). 3. Voir par exemple : Kawashima Yūzō 川島雄三 1962, Shitoyakana kemono しとやかな 獣 (La Bête élégante); Hani Susumu 羽仁進 1963, Kanojo to kare 彼女と彼 (Elle et lui); Nishimura Shōgorō 西村昭五郎 1971, Danchi zuma hiru sagari no jōji 団地妻 昼下りの 情事 (Femme de grand ensemble : affaire d'après-midi); Nakamura Yoshihiro 中村義洋 2013, Minasan, sayonara みなさん、さようなら (Au revoir tout le monde) ; Nakata Hideo 中田秀夫 2013, Kuroyuri danchi クロユリ団地 (Le grand ensemble du lys noir); Koreeda Hirokazu 是枝裕和 2016, Umi yori mo mada fukaku 海よりもまだ深く (Après la tempête); Sakamoto Junji 阪本順治 2016, Danchi 団地 (Grand ensemble). 
permettra de mieux saisir leurs spécificités derrière la patine uniforme des ans, et ainsi de mieux lire le paysage urbain.

Si notre promeneur s'aventure dans les allées et les rues intérieures d'un grand ensemble, il croisera sans doute quelques boutiques aux rideaux métalliques fermés et des passants, ou plus probablement, des passantes, dont l'âge semble à l'unisson de celui des immeubles. Si ce vieillissement commun l'intrigue et qu'il décide de consulter les données du dernier recensement, il se rendra compte que, dans chaque arrondissement de Tokyo, les secteurs où la moyenne d'âge est la plus élevée correspond presque toujours à un ensemble d'habitat public. Comment expliquer ce phénomène? Quelles en sont les conséquences pour les habitants des complexes d'habitat collectif? Voilà deux questions que le promeneur se poserait peut-être et que cet article se propose d'aborder, dans un deuxième et un troisième temps, en s'appuyant de manière conjointe sur les données chiffrées disponibles et sur une ethnographie ${ }^{4}$ conduite pendant quatre ans (2013-2017) dans un grand ensemble d'habitat social du nord-est de Tokyo, à partir de deux points d'observation participante. Le premier m'était offert par le fait que j'y résidais moi-même (décembre 2013-avril 2017) et participais donc à ce titre à la vie collective de l'immeuble (ménage collectif de l'immeuble et de ses alentours, patrouilles de "sécurité", réunions et fête de l'immeuble, etc.). Le second provient du fait que j'ai été pendant un an (mai 2016-avril 2017) membre permanent de l'association d'immeuble. Située au cœur du grand ensemble que j'ai rebaptisé ${ }^{5}$ "Toei Takeshima apāto 都営竹島 アパート» (Appartements Takeshima du gouvernement métropolitain), la barre où je résidais comprend douze étages et un peu plus de deux cent trente logements. Sa construction a été achevée en 1972 et les appartements ont été rénovés dans les années 2000.

4. Cette recherche a pu être conduite grâce au soutien précieux de la Kokusai kōryū kikin 国際交流基金 (Fondation du Japon) et de la Nihon gakujutsu shinkō kai 日本学術 振興会 (Société japonaise pour la promotion de la science).

5. Comme le font souvent les sociologues et anthropologues travaillant sur des terrains "proches» (Pouchelle 1974 : 9; Laé 1985 : 7; Schwartz 2009 : 55-56; Mori 2013; Bestor 1989 : xv), je ne mentionne pas le vrai nom du complexe d'habitat collectif ou l'arrondissement où j'ai mené mon enquête pour protéger l'anonymat des personnes. J'omets ou modifie aussi au besoin des détails qui pourraient permettre d'identifier les lieux ou les individus. 


\section{Politiques publiques de logement et habitat social}

Les différents types de grands ensembles ont une architecture souvent analogue et se retrouvent d'ailleurs parfois au sein du même complexe d'habitat collectif. Si l'on ajoute à cela que leurs dates de construction sont similaires, il est tentant de les ranger dans une même catégorie - de fait, en France, l'appellation d'Habitations à loyer modéré (HLM) inclut aussi des types de logement divers, destinés à des publics de revenus différents, mais la représentation dominante est relativement uniforme. Un retour sur leur histoire permet de faire réapparaître des différences désormais peu visibles si l'on s'arrête aux façades.

La période de l'immédiat après-guerre est marquée par une importante pénurie de logements, avec 4,2 millions d'unités manquantes selon les estimations du gouvernement (Housing Policy Division, Housing Bureau, Ministry of Land, Infrastructure, Transport and Tourism 2016 : 19). Dans ce contexte sont adoptées trois lois qui constituent les trois piliers de la politique de logement de l'après-guerre : la Jütaku kin.yū kōko hō 住宅金融 公庫法 (Loi sur la Caisse nationale de crédit immobilier) en 1950, la Köei jūtaku hō 公営住宅法 (Loi sur les logements publics) en 1951 et enfin la Nihon jūtaku kōdan hō 日本住宅公団法 (Loi sur la Régie japonaise du logement) en 1955 (Hirayama 2006 : 158). La Caisse nationale de crédit immobilier avait pour rôle de soutenir la construction par le biais de prêts à taux bonifiés octroyés aux particuliers ou aux entreprises souhaitant, respectivement, faire construire une maison ou des logements pour leurs employés. La deuxième loi mettait sur pied un système de logements publics dont la construction et l'administration revenaient aux collectivités territoriales (municipalités et départements) et dont le financement provenait du budget national, comme l'explique son article 1 :

Cette loi prévoit la coopération de l'État et des pouvoirs locaux pour fournir les logements nécessaires à une vie saine et civilisée, en proposant des locations ou des souslocations à prix réduits pour les personnes aux bas revenus qui ne sont pas en mesure de trouver un logement, avec pour objectif de contribuer à améliorer la stabilité de la vie des citoyens et au bien-être social (Sumimoto $2012: 5$ ).

La création de la Régie japonaise du logement intervient quatre ans plus tard. Si elle a été traditionnellement présentée comme le «troisième pilier» de la politique du logement de l'après-guerre, Harada Sumitaka 原田純孝(1985) 
montre qu'il a plutôt fallu lui «faire de la place» parmi les dispositifs déjà en place, en durcissant les conditions requises pour l'octroi des prêts à la construction, en diminuant drastiquement le nombre de logements de type 1, destinés aux foyers de revenus faibles à moyens - entre 10000 et 30000 yens mensuels -, et en augmentant ceux de type 2, destinés aux foyers à faibles revenus - moins de 10000 yens mensuels (Ōmoto 1991 : 275).

Les trois piliers de la politique du logement ont donc eu des bénéficiaires socialement différenciés : les personnes à bas revenus (teigaku shotokusha 低額所得者) pour les logements publics des collectivités locales; les classes moyennes pour les logements de la Régie japonaise du logement ${ }^{6}$; les classes moyennes supérieures et les classes supérieures pour les prêts de la Caisse nationale de crédit immobilier. Mais plus qu'une segmentation statique par classes, il s'agissait plutôt, comme l'explique le sociologue urbain Hirayama Yōsuke 平山洋介, d'étapes successives dans ce qui était conçu comme un "parcours de vie» le long d'une "échelle des logements», "de la location d'un logement à l'achat d'une maison, d'un logement de petite taille à un logement plus grand, d'un appartement à un pavillon (Hirayama 2009 : 7-8)».

$\mathrm{Si}$, à un niveau individuel, les logements publics étaient perçus comme une solution transitoire, il en était de même au niveau institutionnel. Comme l'explique le chef du Bureau du logement du ministère de la Construction, Itō Gorō 伊東五郎, devant la commission de la Construction de la Chambre des représentants le 28 février 1950, il s'agissait de «suppléer de façon temporaire» (sono aida no tsunagi その間のつなぎ) à l'offre locative privée le temps qu'elle soit de nouveau capable de répondre à la demande (Hirayama 2009 : 245-246). Et de fait, si l'on compare à d'autres pays où la fourniture de logement public était liée à l'idée de droits sociaux (Marshall 1992), on constate qu'il a toujours occupé une part minime des nouvelles constructions au Japon - moins de $10 \%$ entre 1945 et 1989, sauf entre 1950 et 1959 où cette part atteint 18,4 \%, contre $64 \%$ durant la même décennie en Angleterre ou $46 \%$ en RDA (tableau 1).

6. En 1958, 48,8 \% des résidents de la Régie japonaise du logement gagnaient plus de 40000 yens par mois, contre $32,5 \%$ pour la population active de Tokyo dans son ensemble. Une enquête de février 1960 indique que $75 \%$ d'entre eux étaient employés de bureau ou ingénieurs et $4 \%$ cols bleus contre $30 \%$ et $23 \%$ pour la population active de Tokyo (Keizai kikaku chō 1961 : 140). Ces données sont reprises dans les annexes de l'ouvrage de Laura Neitzel (2016 : 135) sur les danchi. 


\section{TABLEAU 1}

Part (en pourcentage) des logements publics destinés à la location dans l'ensemble des logements construits.

Source : Satō 1999: 304.

\begin{tabular}{|l|c|c|c|}
\hline Année & $\begin{array}{c}\text { Angleterre et Pays de Galles } \\
\text { (logements publics) }\end{array}$ & $\begin{array}{c}\text { Allemagne [RDA] } \\
\text { (logements sociaux) }\end{array}$ & $\begin{array}{c}\text { Japon (logements publics } \\
\text { des collectivités territoriales } \\
\text { et de la régie publique) }\end{array}$ \\
\hline $1945-1949$ & 76,8 & - & 7,4 \\
\hline $1950-1959$ & 64 & 45,8 & 18,4 \\
\hline $1960-1969$ & 43,2 & 28,8 & 9,1 \\
\hline $1970-1979$ & 18 & 19,5 & 7,2 \\
\hline $1980-1989$ & 3,5 & 11,6 & 4,3 \\
\hline
\end{tabular}

De manière plus générale, comme le suggèrent à la fois la chronologie des trois lois et la conception séquentielle d'une «échelle des logements», la promotion de l'accession à la propriété constitue l'axe central de la politique du logement de l'après-guerre (Ronald 2004 : 57). Sous-chef de la section de planification du bureau du logement (ministère de la Construction), Kawashima Hiroshi 川島博 explique dans l'entretien réalisé par Ōmoto Keino 大本圭野 pour l'ouvrage monumental qu'il consacre à la politique japonaise du logement :

Laisser de côté les pauvres? C'est vrai. De toute façon, le nombre de logements était globalement insuffisant. La question était donc de savoir par où on commence. Si l'on avait laissé de côté les gens qui participaient à la reconstruction du Japon en faveur des seules personnes qui allaient être un fardeau, le pays n'aurait pas pu se relever (Ōmoto 1991 : 725-726).

Avec le soutien de la Caisse nationale de crédit immobilier ${ }^{7}$, l'aprèsguerre voit l'avènement progressif d'une «société de propriétaires» (mochi ie shakai 持ち家社会) (Hirayama 2009 : 7-8) : alors qu'avant-guerre, 75,9\%

7. $45,7 \%$ des 51 millions de logements construits depuis la fin de la Seconde Guerre mondiale l'ont été avec des fonds publics (Ronald 2004 : 57). Il s'agit très largement de logements financés par des prêts octroyés par la Caisse nationale de crédit immobilier : 
des logements étaient loués, en 1963, 24,1\% font l'objet d'une location privée, 4,6\% sont des logements publics des collectivités territoriales ou de la Régie japonaise du logement, loués eux aussi, et 64,3\% sont occupés par leurs propriétaires (Hirayama 2009, tableau 1.1). Depuis lors, ce taux s'est maintenu autour de $60 \%$ (Housing Policy Division, Housing Bureau, Ministry of Land, Infrastructure, Transport and Tourism 2016 : 17).

Initié en 1951, l'effort de construction de logements publics atteint un pic en 1971 avec plus de 100000 unités construites, avant de décliner pour passer en dessous de 20000 en 1987, puis de 10000 en 1996 (Housing Policy Division, Housing Bureau, Ministry of Land, Infrastructure, Transport and Tourism 2016 : 27). La Jūtaku kin.yū shien kikō 住宅金融 支援機構 (Agence de soutien financier au logement), qui remplace en 2007 la Caisse nationale de crédit immobilier, cesse de financer directement les prêts pour assumer un rôle de garant pour les emprunts contractés auprès d'institutions bancaires privées. La Régie japonaise du logement devient Jūtaku toshi seibi kōdan 住宅·都市整備公団 (Régie d'aménagement des villes et des logements) en 1981, puis Toshi kiban seibi kōdan 都市基盤 整備公団 (Régie d'aménagement des infrastructures urbaines) en 1999, et enfin, en 2004, sous le gouvernement de Koizumi Jun.ichirō 小泉純一郎 (2001-2006), l'actuelle Toshi saisei kikō 都市再生機構 (Agence de rénovation urbaine, dont le logo est UR pour Urban Renaissance) après sa fusion avec la division d'une autre entité administrative. L'ancienne régie publique devient ainsi une dokuritsu gyōsei hōjin 独立行政法人, une entité juridique administrative indépendante semi-publique, dont le cœur d'activité n'est plus la construction de logements publics mais l'aménagement urbain et la gestion d'un parc vieillissant de 746213 logements en 2015 (Sera 2015 : 2). Dans le premier domaine, à l'instar de l'Agence de soutien financier au logement, son action n'est désormais plus directe mais se limite à soutenir des projets de redéveloppement urbain menés par des acteurs privés (Sato 2007 : 87). Dans le second domaine, l'Agence a «cessé de construire de nouveaux logements destinés à la location, vend tout ce qu'il est possible de vendre de son parc de logements au secteur privé et externalise chaque fois qu'elle le peut la gestion des immeubles restants à des entreprises privées»

49,5 \% pour la période 1961-1965 et plus de 75,6 \% depuis 1976 (Hirayama 2003 : 144). 
(Sato 2007 : 87). Elle rénove, reconstruit ou détruit progressivement les complexes d'habitat collectif, en les adaptant aux besoins de résidents vieillissants (ascenseurs, accessibilité aux chaises roulantes, etc.). La construction de nouveaux logements publics par les collectivités territoriales connaît elle aussi une baisse rapide à partir du début des années 1970 pour atteindre un niveau quasi nul au début des années 2000 (Hirayama 2006 : 173). Parallèlement, les réformes successives de la Loi sur le logement public, en abaissant le plafond de revenus, en restreignent l'accès aux très bas revenus. $\mathrm{Si}$, en $1951,80 \%$ de la population ${ }^{8}$ pouvait prétendre à un logement public des collectivités territoriales, ce chiffre passe à $33 \%$ dans les années 1970 puis, avec la révision de 1996, à $25 \%$ (Hirayama 2006 : 228). À l'heure actuelle, à Tokyo, le plafond de revenus est, dans le cas général ${ }^{9}$, de 189666 yens par mois pour un foyer de deux personnes (Tōkyōto jūtaku kyōkyū kōsha : 1). Dans les logements gérés par l'Agence de rénovation urbaine, il n'y a pas de plafond de ressources mais un revenu plancher de quatre fois le loyer ou 330000 yens mensuels ${ }^{10}$ (UR Toshi saisei kikō 2017). L'attribution des logements se fait par tirage au sort pour les logements des collectivités territoriales et sur le principe du "premier arrivé premier servi » pour ceux de l'Agence de rénovation urbaine.

Pour être exhaustif, il faudrait préciser que le gouvernement de Tokyo gère désormais deux autres types de logements publics, les ippan chintai jütaku 一般賃貸住宅 (logements locatifs ordinaires) et les tomin jütaku 都民住宅 (logements à destination des Tokyoïtes). Les premiers sont ouverts à tous, célibataires et familles, à partir d'un revenu plancher déterminé en fonction du loyer, comme pour les logements UR, et sans possibilité de subventions. Les seconds sont réservés principalement aux familles et peuvent bénéficier d'un loyer subventionné selon la tranche de revenus du foyer, définie par un revenu plancher et un revenu plafond, le revenu plancher

8. Ce chiffre ne prend en compte que le critère du revenu. D'autres conditions, ayant trait à la composition du foyer et la nationalité, s'ajoutaient aussi à l'origine, excluant les célibataires et les étrangers.

9. Comme on le verra plus loin, le plafond de revenus est plus élevé pour certaines catégories spécifiques, comme les personnes âgées de plus de 60 ans ou les foyers comprenant des personnes souffrant de handicaps.

10. Pour les appartements d'un loyer supérieur à 200000 yens, le revenu plancher est de 400000 yens. 
de la tranche inférieure (I-1) s'élevant à 231666 yens par mois pour un foyer de deux personnes et le revenu plafond de la tranche supérieure $(\mathrm{V})$ à 632666 yens par mois. Ce type de logements s'adresse notamment aux foyers dont les revenus, bien que peu élevés, se trouvent, depuis la réforme de la Loi sur les logements publics de 1996, au-dessus du plafond de revenus pour les toei jütaku'11.

Revenir sur la politique du logement mise en place dans l'après-guerre a permis de faire apparaître, entre les logements des collectivités territoriales et ceux de la Régie japonaise du logement (devenue Agence de rénovation urbaine), des différences pérennes - dont témoignent les conditions d'accès actuelles -, et ce, en dépit de leur histoire commune, de leur fréquente proximité spatiale et de leur ressemblance architecturale. Ces deux types de grands ensembles partagent aussi une autre caractéristique : l'âge élevé de leurs habitants.

\section{2. Âge du bâti, âge des habitants ? Grands ensembles publics et vieillissement}

\subsection{Les grands ensembles publics, quartiers urbains les plus âgés}

Au début des années 2000, la question du vieillissement dans les grands ensembles a fait une entrée fracassante dans l'espace médiatique avec les reportages sur les morts solitaires (kodoku shi 孤独死). En 2001, le squelette d'un homme de 69 ans est retrouvé, trois ans après sa mort, dans un petit appartement de Tokiwa daira danchi 常盤平団地 (1960, Toshi saisei kikō, 4839 appartements, ville de Matsudo, département de Chiba). Comme il avait choisi le prélèvement automatique pour le paiement de son loyer et de ses factures, sa banque a continué à effectuer les paiements correspondant au loyer et aux factures de gaz, d'électricité et d'eau, jusqu’à épuisement de son compte. Après que les courriers de mise en demeure lui sont revenus, le responsable du dossier au bureau de l'Agence de rénovation urbaine

11. Il reste cependant, de manière un peu surprenante, un trou entre les deux dispositifs concernant, pour les foyers de deux personnes, ceux qui touchent plus de 189666 yens et moins de 231666 yens par mois. 
de Matsudo s'est rendu à l'appartement de la personne puis a contacté la police, qui a découvert le squelette (Nakazawa 2008 : 3-4). L'année suivante, une autre personne est retrouvée à Tokiwa daira danchi, trois mois après sa mort : entre 2002 et 2005, on y dénombre ainsi vingt-et-un cas de morts solitaires (Nippon hōsō kyōkai 2005).

Durant la seconde moitié des années 2000, le problème des morts solitaires est réinscrit par les médias dans la thématique plus large des genkai danchi 限界団地 (grands ensembles seuils) (Asahi Shinbun 2009), déclinaison urbaine des genkai shüraku 限界集落 (villages seuils) dont la vie communautaire est mise en péril par la dépopulation et son corrélat, le vieillissement. Pour l'inventeur du concept, Ōno Akira 大野晃 (2005, 2008), un village devient «village seuil» quand la proportion des résidents de plus de 65 ans dépasse $50 \%$ et les grands ensembles seuils sont définis de la même manière. Toei Toyama haitsu apāto 戸山ハイツアパート (19681976, 3019 appartements, Shinjuku) a ainsi eu les honneurs réguliers des médias (Watanabe 2015 : 29).

Au-delà du traitement médiatique ponctuel de ces thématiques, le vieillissement des résidents des grands ensembles de Tokyo rejoint une tendance démographique de fond du début du $\mathrm{XxI}^{\mathrm{e}}$ siècle $^{12}$ pour laquelle il constitue un miroir grossissant. Les données du recensement de 2010 (Sōmushō tōkei kyoku 2010) montrent ainsi que, dans les arrondissements où il y a de grands ensembles ${ }^{13}$, ceux-ci correspondent presque toujours aux districts (chōme 丁目 ${ }^{14}$ ) où la population est la plus âgée. Si en 2010 les plus de 65 ans représentaient $20 \%$ de la population du département de Tokyo (Tōkyō-to), à Kita-ku par exemple, ils sont les plus nombreux à Kirigaoka

12. En octobre 2015, les plus de 65 ans constituaient $26,7 \%$ de la population (Naikaku fu $2016: 2$ ).

13. À Meguro-ku, le complexe d'habitat municipal le plus grand, les Toei Meguro itchōme apāto 都営目黒一丁目アパート, ne comprend que cinq immeubles de cinq étages et deux cent dix-sept appartements (Meguro Kuyakusho 2016). Dans ce type de cas, les données par districts ne sont pas assez précises car les immeubles de logement public n'en occupent qu'une partie très réduite - moins d'un dixième dans ce cas précis. De fait, l'arrondissement compte peu de toei jūtaku: huit en tout, dont la moitié de moins de quarante logements.

14. Les chōme constituent les plus petites unités administratives urbaines désignées par le nom du $c h \bar{o} 丁$ associé au numéro de la subdivision. 
itchōme 桐ヶ丘 1 丁目 $(51,89 \%)$, où se trouve l'ensemble d'habitat collectif Tōei Kirigaoka apāto, et à Akabanedai itchōme 赤羽台 1 丁目 (51,12 \%), qui est l'adresse d'Akabanedai danchi, tous deux évoqués en introduction. Le même phénomène se retrouve dans les autres arrondissements de la ville, comme l'illustre le tableau 2 ci-dessous. S'il existe des différences selon les arrondissements, la proportion de plus de 65 ans dans les districts les plus âgés dépasse en général $35 \%$ - leur nombre et leur part relative ont d'ailleurs augmenté depuis 2010.

\section{TABLEAU 2}

District le plus âgé de l'arrondissement (en pourcentage des plus de 65 ans) et ensemble d'habitat social correspondant (liste partielle).

\begin{tabular}{|c|c|c|}
\hline Arr. & District le plus âgé & Ensemble d'habitat social correspondant \\
\hline \multirow[t]{2}{*}{ Kita } & $\begin{array}{l}\text { Kirigaoka itchōme } \\
\text { 桐ヶ丘 } 1 \text { 丁目 } \\
\mathbf{5 1 , 8 9 \%}\end{array}$ & Toei Kirigaoka apāto 都営桐ケ丘アパート \\
\hline & $\begin{array}{l}\text { Akabanedai itchōme } \\
\text { 赤羽台 1 丁目 } \\
\mathbf{5 1 , 1 2 \%}\end{array}$ & Akabanedai danchi 赤羽台団地 \\
\hline \multirow[t]{3}{*}{ Shinjuku } & $\begin{array}{l}\text { Kasumigaoka machi } \\
\text { 霞ヶ丘町 } \\
\mathbf{4 9 , 8 9 \%}\end{array}$ & Toei Kasumigaoka apāto 都営霞ヶ丘アパート15 \\
\hline & $\begin{array}{l}\text { Hyakuninchō yon chōme } \\
\text { 百人町 } 4 \text { 丁目 } \\
\mathbf{4 5 , 0 6 \%}\end{array}$ & $\begin{array}{l}\text { Toei hyakuninchō yon chōme dai-ichi apāto } \\
\text { 都営百人町四丁目第一アパート } \\
\text { Toei hyakuninchō yon chōme dai-ni apāto } \\
\text { 都営百人町四丁自第二アパート } \\
\text { Toci hyakuninchō yon chōme dai-san apāto } \\
\text { 都営百人町四丁目第三アパート }\end{array}$ \\
\hline & $\begin{array}{l}\text { Toyama ni chōme } \\
\text { 户山2 } 2 \text { 丁目 } \\
\mathbf{4 3 , 1 9 \%}\end{array}$ & Toei Toyama Haitsu apāto 都営戸山ハイツアパート16 \\
\hline Setagaya & $\begin{array}{l}\text { Ōkura san chōme } \\
\text { 大蔵 } 3 \text { 丁目 } \\
\mathbf{4 3 , 6 6 \%}\end{array}$ & Kōsha Ōkura jūtaku 公社大蔵住宅 \\
\hline Minato & $\begin{array}{l}\text { Kita Aoyama san chōme } \\
\text { 北青山 } 3 \text { 丁目 } \\
\mathbf{4 1 , 5 4 \%}\end{array}$ & Toei Kita Aoyama Apāto 都営北青山アパート \\
\hline Adachi & $\begin{array}{l}\text { Take no tsuka nana chōme } \\
\text { 竹の塚 } 7 \text { 丁目 } \\
\mathbf{3 9 , 9 5 \%}\end{array}$ & Toei Take no tsuka nana chōme apāto 都営竹の塚 7 丁目アパート \\
\hline
\end{tabular}

15. Cet ensemble d'habitat collectif, où $56,32 \%$ des femmes ont plus de 65 ans, va être rasé pour permettre l'agrandissement du Kokuritsu kyōgijō 国立競技場 (Stade national) (Inaba 2015).

16. D'après ces chiffres-là, peut-être moins précis que ceux dont disposaient les médias sur le seul complexe d'habitat de Toyama Haitsu, ce grand ensemble n'est pas encore un genkai danchi. 
$\mathrm{Si}$, comme on vient de le voir, les grands ensembles présentent une population nettement plus âgée que celle de Tokyo, parmi eux, la proportion des plus de 65 ans est plus élevée dans les toei jūtaku. Pourquoi?

\subsection{Le vieillissement comme artefact démographique}

Comme les villes nouvelles, les grands ensembles sont à l'origine des artefacts démographiques définis notamment par un "déséquilibre des structures initiales des âges (Le Bras 1976 : 274)», avec «beaucoup d'enfants, peu d'adolescents et de très jeunes adultes, beaucoup d'adultes entre 25 et 45 ans, très peu de vieux» (Le Bras 1976 : 271). On pourrait ainsi les qualifier, en France comme au Japon, de "quartiers à deux générations ", comme on a parlé pour les villes nouvelles de "villes à deux générations» (P. 1960 : 138). Ce déséquilibre des structures d'âge induit une série d'effets parfois sensibles seulement à très long terme.

Les plus immédiatement visibles concernent les équipements urbains : les écoles, aux petites classes surchargées durant les années qui suivent l'installation initiale des résidents ${ }^{17}$, se vident progressivement, à mesure que cette vague d'enfants gravit les échelons scolaires, avant de fermer ou de fusionner avec une autre école du quartier. Dans le complexe d'habitat public de Takeshima, l'école primaire située en dessous de notre immeuble a fermé au début des années 1990, peu après que la kodomokai 子供会 (association des enfants) organisée par la jichikai 自治会 (association des résidents) de l'immeuble eut cessé ses activités. À la fin de la décennie, le président précédent de la jichikai a décidé de créer une fukujukai 福寿会 (association pour une vie longue et heureuse). Le remplacement de la kodomokai par une fukujukai résume assez bien l'itinéraire démographique de nombre de grands ensembles de Tokyo : les enfants des nouveaux résidents ont grandi et quitté pour la plupart le domicile des parents, ce qui entraîne mécaniquement une diminution et un vieillissement de la population. Âgés

17. Et ce d'autant plus qu'avant la réforme de la Köei jūtaku hō de 1980, les célibataires ne pouvaient pas se porter candidats pour un logement (Kōei jūtaku seido kenkyū kai $2006: 70)$. 
de 20 à 40 ans lors de leur installation, les parents en ont maintenant 35, 40, voire 50 de plus pour les ensembles d'habitat les plus anciens. Les taux de plus de 65 ans cités plus haut témoignent de l'évolution de cette pyramide des âges déséquilibrée dont les cohortes les plus nombreuses se sont déplacées vers le sommet. Les différents modèles statistiques élaborés par Hervé Le Bras et Jean-Claude Chesnais suggèrent qu' «après un extrême vieillissement, de nombreux logements sont libérés et la population se rajeunit et augmente un peu» $(1976$ : 282). De fait, cette évolution se constate dans l'immeuble où j'habitais, qui comptait dix-huit logements vacants en avril 2017. Chaque année, meurt un petit nombre de résidents : les permanents de l'association d'immeuble chargés de la comptabilité, comme c'était mon cas entre mai 2016 et avril 2017, l'apprennent lors de la collecte des cotisations mensuelles ou, comme tout le monde, par les avis parfois placés sur le panneau d'affichage du rez-de-chaussée. D'autres quittent l'immeuble pour une maison de retraite médicalisée. Après réfection, les appartements sont alors réattribués par tirage au sort deux fois par an. Les nouveaux arrivants sont pour partie des couples avec ou sans enfants. Après une période où ils étaient absents, quarante enfants vivent désormais dans l'immeuble : le président de l'association d'immeuble a profité des animations organisées le jour d'Halloween (31 octobre 2016) pour les photographier, et les compter. Durant les années qui ont suivi l'installation des premiers résidents, dans les années 1970, il y en avait, raconte-t-il, plus de deux cents.

Plus marqué que celui des autres secteurs urbains, le vieillissement des habitants des grands ensembles se justifie donc en partie par des structures démographiques fortement déséquilibrées, héritées de leurs modalités de peuplement. Elles n'expliquent pas cependant pourquoi, dans presque tous les arrondissements, les zones où les résidents âgés sont les plus nombreux sont des ensembles de toei jütaku, et non des complexes construits par la Régie japonaise du logement, souvent à la même époque. Un autre facteur doit être pris en compte, qui a trait cette fois aux évolutions des politiques de logement public.

\subsection{Le vieillissement comme artefact politique}

Les politiques de logement public kōei jūtaku sont conduites, on l'a vu, par l'État et les collectivités territoriales, c'est-à-dire, pour la capitale, le 
gouvernement métropolitain de Tokyo. Si, au niveau national, les révisions de la loi de 1951 et les décrets d'application successifs en constituent autant d'inflexions, pour Tokyo, un tournant majeur a lieu au mitan des années 1990. Alors que la spéculation foncière avait entraîné une hausse des loyers, le gouvernement métropolitain adopte une politique volontariste de logement, avec un premier plan d'action en 1991 (Tōkyōto jūtaku kyōkyū sōmu bu kikaku shitsu 1991). Mais les ressources disponibles diminuent avec l'éclatement de la bulle et la récession. Adopté pendant le premier mandat (1999-2003) du gouverneur Ishihara Shintarō 石原慎太郎, le plan d'action de 2002 (Tōkyōto jūtaku kyōkyū sōmu bu jūtaku seisaki shitsu 2002) s'en remet au marché pour satisfaire aux besoins de logement, les toei jütaku ayant vocation à former un «filet de sécurité» pour les secteurs les plus pauvres (Hirayama 2006 : 227). Dans ce contexte, comme indiqué déjà plus haut, la construction de nouveaux logements est quasiment nulle et les projets de rénovation bénéficient surtout aux résidents déjà logés (Watanabe 2015 : 30). Concrètement, cela implique que le déséquilibre des structures démographiques à l'échelle des districts n'est pas transformé par l'arrivée de nouveaux résidents dans les immeubles, comme dans d'autres quartiers où les arrivées ne font pas l'objet de régulations.

Les révisions de la Loi sur le logement public et les décrets d'application successifs adoptés au niveau national ont même eu l'effet inverse. La révision de 1980 permet l'accès aux logements publics de personnes célibataires âgées de plus de 50 ans, pour les femmes, et de plus de 60 ans, pour les hommes (Kōei jūtaku seido kenkyū kai 2006 : 70). D’un point de vue strictement démographique, la mesure aurait été pertinente lors de l'adoption de la loi en 1951, en permettant d'intégrer aux résidents une classe d'âge alors inexistante et de pallier le déséquilibre de la pyramide des âges (P. 1960 : 138). Mais en 1980, cette mesure renforce au contraire la classe déjà surreprésentée des seniors en devenir. Le passage de l'âge plancher à 60 ans en 2005, après son unification à 50 ans sans distinction de sexe en 1996 (Kōei jūtaku seido kenkyū kai 2006 : 78-79) ne fait que consolider cette tendance.

C'est aussi le cas pour d'autres dispositions prises en 1996 et 2005. À l'heure actuelle, le plafond de revenus mensuels, abaissé en 1996 pour le cas général (à l'exclusion des plus de 60 ans) est de 189666 yens pour un foyer de deux personnes de moins de 60 ans et de 245667 yens pour un foyer de deux personnes dont l'une de plus de 60 ans (Tōkyōto jūtaku kyōkyū kōsha 2017 : 1). 
La révision de 2005 a mis en place des baux à durée limitée de 10 ans pour les moins de 40 ans. En cas de dépassement durable d'un plafond de revenus, les résidents doivent en outre s'acquitter d'un loyer équivalent à ceux du secteur privé dans le quartier et sont invités à quitter leur logement (Watanabe 2015 : 30). Cela concerne là encore surtout les résidents moins âgés et toujours actifs.

Bénéficiant d'un plafond de revenus plus élevé, les plus de 60 ans sont donc statistiquement plus nombreux, non seulement à pouvoir faire une demande de logement, mais aussi à en obtenir un. Les moins de 60 ans, à l'inverse, y accèdent moins facilement, pour une durée limitée et sous conditions de ressources. Ces dispositions ont pour effet démographique d'augmenter la classe d'âge située au sommet de la pyramide et contribuent ainsi au vieillissement observé dans le recensement de 2010. Le vieillissement est bien aussi un artefact politique.

Le type de peuplement initial des immeubles et l'évolution des conditions d'accès aux logements sociaux concourent donc à construire le vieillissement prononcé de leur population. Artefact politique autant que démographique, le vieillissement n'en est pas moins une réalité palpable au quotidien dans les grands ensembles. Quels en sont les effets sur l'organisation de la vie collective?

\section{Seniors, jeunes et marché : l'organisation de la vie collective face au vieillissement}

Au Japon, les associations de quartier occupent depuis longtemps une place importante dans la vie quotidienne ${ }^{18}$. Présentes sur l'ensemble du territoire national, ces quelques 300000 associations se caractérisent par un taux élevé de participation, au moins nominale et financière, des habitants du district rural ou urbain qui en relève (Tsujinaka 2014 : 1, 3). Leurs fonctions, diverses, varient selon les lieux. La première enquête nationale

18. Selon les lieux et leur histoire, ces associations sont nommées tour à tour jichikai 自治会 (association d'autogestion), chōnaikai 町内会 (association interne au quartier), chōkai 町会 (association de quartier), kukai 区会 (association de secteur), burakukai 部落会 (association de hameau)... 
conduite entre août 2006 et février 2007 confirme toutefois que la majorité d'entre elles prend en charge la gestion des déchets (69\% des associations), l'organisation de fêtes $(74 \%)$ et d'événements sportifs et culturels (64\%), organise des activités en lien avec la prévention des catastrophes naturelles (55\%), des incendies (59\%) et de la criminalité (52\%), ainsi qu'avec la sécurité routière (53\%). Elles s'occupent de l'entretien des rues et de l'éclairage $(86 \%)$, font circuler des informations propres au quartier $(62 \%)$ ou transmises à cette fin par la municipalité, soutiennent le travail éducatif des écoles (63\%), prennent enfin des initiatives en faveur des jeunes (52\%) et des seniors (70\%) (Tsujinaka $2014: 210-212)$.

Dans les grands ensembles, dont la logique territoriale diffère de celle des quartiers plus anciens, ces associations sont en général nommées jichi$k a i^{19}$ et sont relativement actives, pour les mêmes raisons qui expliquent le vieillissement : un nombre important de résidents vit sur place depuis longtemps et les jichikai créées au moment de leur installation continuent à fonctionner, dans les grandes lignes, suivant les principes adoptés au départ, même si l'augmentation de la moyenne d'âge entraîne aussi une évolution des besoins. La permanence du bâti va de pair avec la permanence des structures collectives principales.

En termes de fonctionnement, ces associations d'immeuble(s) ne souffrent pas à première vue de la présence de résidents âgés, au contraire : les activités des permanents de l'association - dont les mandats, parfois renouvelés, durent d'un à quatre ans - requièrent d'avoir du temps et, de fait, les retraités y occupent souvent une place prépondérante - $44 \%$ des personnes impliquées selon l'enquête de $2006-2007^{20}$, pour un âge moyen des présidents d'association de 65 ans (Tsujinaka 2014:212, 221).

Mais la présence importante des seniors est un effet du vieillissement, plus que du temps libre. Une grande partie des seniors continue en effet à travailler, souvent à plein temps. Dans l'immeuble où je vivais, qui constitue une jichikai à lui seul, les trois principaux groupes de permanents sont

19. Suivant les cas, les jichikai peuvent se limiter à un seul immeuble ou en englober plusieurs, en fonction du nombre d'étages et de la taille des bâtiments mais aussi des singularités historiques. De fait, même si elles réalisent des activités similaires, leur organisation n'est jamais complètement identique et les mêmes fonctions pourront être nommées différemment.

20. La proportion des employés à plein temps est de $20 \%$. 
respectivement les honbu yakuin 本部役員 (membres du comité directeur) - fonction qui prend le plus de temps, avec au moins deux réunions par mois -, les kakukai yakuin 各階役員 (représentants des étages) et les bōsai bōhan yakuin 防災防犯役員 (chargés de la prévention des sinistres et des délits). Sur les onze membres du comité directeur, dont j'ai fait partie entre mai 2016 et avril 2017, trois avaient moins de 45 ans et les huit autres plus de 65 ans, souvent même plus de 70 ans : on retrouve là aussi le creux dans la structure des âges évoqué plus haut, avec l'absence de classe d'âge intermédiaire. Sur les huit seniors, seuls le président de l'association et l'une des quatre femmes ne travaillaient pas.

Un autre signe témoigne du fait que la moyenne d'âge élevée des permanents est bien un effet du vieillissement : le rapport des résidents et, plus particulièrement, des permanents de la jichikai avec les nouveaux arrivants jeunes. Les habitants de l'immeuble se réjouissent de voir arriver à leur étage des couples jeunes, souvent avec des enfants, plutôt que d'autres seniors. On leur demande aussi très vite d'assumer l'une des trois charges principales de l'association, en devenant membres du comité directeur (deux ans), représentants des étages (un an) ou chargés de la prévention des sinistres et des délits (un an). Dans l'immeuble où j'avais emménagé en 2013, tous les hommes des familles arrivées la même année ou les années suivantes ont accepté une charge, souvent immédiatement. Les charges se transmettent toujours en avril, à l'occasion de la sōkai 総会 (assemblée générale). On devient en général d'abord représentant des étages ou chargé de la prévention des sinistres et des délits. En avril 2016, trois des jeunes arrivants des années précédentes, dont je faisais partie, sont devenus membres $\mathrm{du}$ comité directeur et un quatrième a assumé la charge de fuku buchō 副 部長 (vice-directeur) du groupe des chargés de la prévention des sinistres et des délits. Les permanents de la jichikai ont conscience que l'association et, à travers elle, l'immeuble, a besoin de «forces vives " pour que la jichikai continue à exister et à remplir son rôle.

En charge de la gestion du logement public municipal, la Tōkyō-to jūtaku kyōkyū kōsha 東京都住宅供給公社 (Régie de fourniture de logements du gouvernement métropolitain de Tokyo, JKK) trouve en la jichikai une interlocutrice précieuse en même temps qu'une gestionnaire très économique de ses biens immeubles. Elle semble prendre au sérieux elle aussi les menaces que représente le vieillissement et mesurer l'importance 
de la participation des jeunes résidents. Selon le président de la jichikai ${ }^{21}$, les employés de la JKK, interrogés sur ce point, ont ainsi indiqué lors d'une réunion que les jeunes résidents impliqués dans l'association de manière durable - et non pour un an seulement - pourraient obtenir la prolongation de leur contrat au-delà des dix ans prévus.

Une autre piste explorée pour diminuer le poids des tâches collectives consiste à repenser l'organisation de la vie collective. Trois membres du comité directeur sont par exemple chargés d'ouvrir et de fermer deux fois par semaine les différents espaces destinés au dépôt des ordures et des déchets recyclables (canettes, verres, papiers et cartons). L'emplacement extérieur pour les ordures est ouvert le soir de $18 \mathrm{~h}$ à $22 \mathrm{~h}$, puis de nouveau le matin, jusqu'au passage du camion poubelle vers $13 \mathrm{~h}$. Mais cette tâche apparaît désormais un peu lourde pour les permanents qui ont tous près de 70 ans. Le projet en discussion lors des réunions début 2017 était donc de fournir une clef par foyer - comme dans d'autres grands ensembles - pour que les résidents puissent jeter leurs poubelles quand bon leur semble, libérant ainsi les permanents de cette tâche. Ce genre de détail apparemment insignifiant est représentatif de la multitude de défis minuscules que le vieillissement impose à la vie collective ${ }^{22}$.

Une troisième piste, aux répercussions plus grandes sur l'organisation de la vie collective, est d'externaliser des tâches jusque-là accomplies par la communauté des résidents ou par les permanents. À Takeshima, le ménage des parties communes est divisé en quatre volets. Chaque foyer prend en charge le ménage des espaces poubelles pendant une semaine, avant de passer les clés et le carnet de suivi à l'appartement suivant. Le ménage des parties communes de l'étage, au niveau de la sortie d'ascenseur et des trois escaliers, se fait à tour de rôle, les résidents concernés assumant des responsabilités tournantes d'un mois - soit quatre ménages hebdomadaires - ou d'une semaine, selon une organisation propre aux étages. Le ménage autour de l'immeuble, qui a lieu en général le troisième dimanche du mois à $8 \mathrm{~h} \mathrm{du}$

21. Explication du président de l'association destinée aux résidents jeunes assumant la charge de honbu yakuin à la fin du repas partagé qui a suivi le réaménagement de la salle de réunion rénovée, dimanche 15 janvier 2017 (journal de terrain).

22. L'expression qui revient régulièrement dans les réunions de permanents en lien avec les différentes tâches à effectuer est : «taihen desu 大変です» (c’est dur). 
matin, est assuré en alternance par les étages 2 à 6 et 7 à 12, les différentes zones faisant l'objet d'une répartition elle aussi tournante entre les étages. Très important le lendemain des jours de pluie, le ménage devant l'ascenseur et les boîtes aux lettres au rez-de-chaussée, autrefois effectué à tour de rôle, est confié depuis quelques années à une entreprise. Mais, à cause du coût important et du manque de flexibilité - la pluie ne tombe pas à jour fixe -, il a été décidé lors de l'assemblée générale du 23 avril 2017 que le ménage serait à nouveau confié à des résidents : une ou deux personnes «bénévoles» qui recevront une rémunération compensatoire.

Tous les foyers ne participent pas au ménage du dimanche matin, certains, en raison de leur condition physique, d'autres, sans excuse considérée valable par les autres résidents de l'étage, ce qui entraîne des discussions récurrentes sur les mesures à prendre (amende compensatoire...). Au deuxième étage, où la moyenne d'âge est la plus élevée, la part de ménage a été confiée à une entreprise. De même, lors de l'assemblée générale d'avril 2017, il a été décidé d'externaliser la gestion des éclairages de l'immeuble, jusque-là assurée par les chargés de la prévention des sinistres et des délits. Dans le cadre d'un bail de 6 ans, l'entreprise contractante s'engage à remplacer tous les néons traditionnels par des modèles à diode électroluminescente (LED), qui consomment moins, et à venir les remplacer le cas échéant, pour 20900 yens par mois. Lors des réunions de permanents ${ }^{23}$, le principal argument en faveur, outre les économies d'électricitée ${ }^{24}$ est le fait que changer les néons en utilisant un escabeau sur les coursives est dangereux, particulièrement pour des personnes âgées et des femmes (sic).

Comme le montrent ces différents exemples, une partie des tâches assumées par la jichikai de Takeshima est progressivement externalisée de la sphère de l'autogestion et de la participation volontaire et gratuite vers la sphère marchande - avec, à terme, le spectre d'une disparition de la jichikai. Si les activités les plus matérielles seraient déléguées, une partie des fonctions qu'elle assure, de manière formelle - divers événements organisés - et informelle, en tant que pièce maîtresse de la vie collective locale - maintien d'un contact avec l'ensemble des résidents, présence de proximité et

23. Réunions mensuelles des membres du comité directeur, le 7 avril 2017 et des jōnin.iin 常任委員 (permanents), le 14 avril 2017.

24. La JKK devait cependant installer des modèles à LED dans un avenir proche. 
médiation en cas de conflits - sont plus difficilement transférables. Et les conséquences négatives d'une vie communautaire affaiblie touchent particulièrement les plus âgés - isolement, démence sénile et mort solitaire (Watanabe 2015 : 29 30).

Le vieillissement fait ainsi obstacle à la poursuite des fonctions de la jichikai, alors qu'il les rend précisément plus nécessaires. Si, dans d'autres grands ensembles, les tâches ont été externalisées, à Takeshima, la disparition de la jichikai sert surtout pour l'instant de menace, lors des réunions par étage auxquelles donnent lieu les patrouilles de sécurité d'hiver, pour inciter les résidents à accepter des charges de permanents l'année suivante. La menace a aussi une dimension financière car la délégation au secteur privé peut tripler les charges mensuelles ${ }^{25}$. La fragilisation des associations de quartier est une réalité nationale, au-delà des seuls grands ensembles (Nippon hōsō kyōkai 2015; Ōno 2008). Pour les complexes d'habitat social ${ }^{26}$, l'arrivée de résidents plus jeunes et leur intégration au sein de la jichikai peuvent constituer un chemin d'avenir, comme en ont clairement conscience les permanents plus âgés mais aussi la JKK.

Pour faire un tour d'horizon de la situation actuelle des grands ensembles d'habitat public de Tokyo, ce texte s'est donc proposé d'articuler une mise en perspective historique à une focalisation sur la question du vieillissement. Dans un premier temps, revenir sur la politique japonaise du logement initiée dans les années 1950 a permis de faire apparaître les spécificités des différents types de logement public, toujours présentes malgré les transformations des organismes gestionnaires et des modes de gestion. Plafonds et planchers de revenus témoignent de la persistance de la distinction entre les logements publics administrés par les collectivités territoriales (destinés aux personnes aux revenus les plus modestes) et par la Régie japonaise du logement, devenue Agence de rénovation urbaine (destinés aux classes

25. Le 17 décembre 2016, le trésorier de l'association expliquait que si la jichikai cessait d'assumer ses tâches actuelles, les charges mensuelles passeraient de 1500 yens par foyer à environ 5000 yens, comme dans d'autres grands ensembles (journal de terrain et enregistrement vidéo).

26. Et au-delà aussi : des municipalités rurales mettent ainsi en place des politiques incitatives à l'installation de résidents jeunes. En tant que bailleur, la JKK dispose cependant de moyens d'action beaucoup plus puissants. 
moyennes), au-delà du vieillissement commun de leurs parcs locatifs. Ce vieillissement touche aussi les résidents. Dans le cas des logements publics municipaux, où les taux de plus de 65 ans sont les plus élevés, il s'agit d'un artefact politique autant que démographique. Mais c'est aussi une réalité tangible qui affecte notamment l'organisation de la vie collective, comme le montre l'exemple de Takeshima : les associations d'immeubles tentent d'y faire face en intégrant autant que possible les arrivants jeunes, en réorganisant ou en externalisant certaines tâches. Même si les modélisations des démographes en indiquent déjà les perspectives générales - vieillissement extrême suivi d'un rajeunissement et d'une augmentation du nombre des résidents -, il serait intéressant de connaitre l'évolution de la situation des grands ensembles municipaux à la fin des années 2020, d'autant qu'il est probable que les problèmes mentionnés entraînent de nouvelles inflexions de la politique de logement public. 


\section{Bibliographie}

Ne sont mentionnées ici que les références citées dans le corps de l'article.

\section{Romans, magazines, mangas, documentaires télévisés et livres non académiques}

IMAI Tetsuya 今井哲也 2011a

Bokura no yoake 1 ぼくらのよあけ. 1 (Notre aube), Tokyo, Kōdansha 講談社, 224 p.

IMAI Tetsuya 今井哲也 2011b

Bokura no yoake 2 ぼくらのよあけ. 2 (Notre aube), Tokyo, Kōdansha 講談社, 252 p.

HASEDA Ippei 長谷田一平 (éd.) 2015 Foto ākaibu shōwa no Kōdan jūtaku: danchi shinbun no kishatachi ga kiroku shita sokuseki フォトアーカイブ昭和の 公団住宅: 団地新聞の記者たちが記録し た足跡 (Archives photo de la régie du logement), Tokyo, Chishobō 智書房, $211 \mathrm{p}$.

ISHIMOTO Kaoru 石本馨 2008

Danchi junrei: Nihon no unda kiseki no jūtaku yōshiki 団地巡礼 : 日本の生んだ奇跡 の住宅様式 (Pèlerinage dans les grands ensembles : les prodigieux styles de logement que le Japon a engendré), Tokyo, Futami Shobō 二見書房, 192 p.

Ms 2011a

Shōjo kōdan apātomento 1 少女公団 アパートメント 1 (Les filles des grands ensembles 1), Tokyo, Hōbunsha 芳文社, $118 \mathrm{p}$.
Ms 2011b

Shōjo kōdan apātomento 2 少女公団 アパートメント 2 (Les filles des grands ensembles 2), Tokyo, Hōbunsha 芳文社, $118 \mathrm{p}$.

Nippon hōsō kyōkai 日本放送協会 2005 «Hitori danchi no isshitsu de»ひとり団地 の一室で (Seul dans un appartement de grand ensemble), NHK supesharu NHK Z ペシャル, 24 septembre, 52 minutes.

Nippon hōsō kyōkai 日本放送協会 2015 "Chōnaikai ga kieru ? Dō suru chiiki no tsunagari»町内会が消える? 〜 ど うする地域のつながり〜 (Disparition des associations de quartier? Que deviendraient les liens locaux?), NHK kurōzu appu gendai purasu NHK クローズ アップ現代+， 4 novembre, 26 minutes.

ODA Tobira 小田扉 2004

Danchi Tomō 団地ともお 1 (Tomo: une enfance dans un grand ensemble), Tokyo, Shōgakukan 小学館 (le tome 29 est sorti en avril 2017), 185 p.

ŌYAMA Ken 大山顕 2008

Danchi no kenkyū 団地の見究 (Étude des grands ensembles), Tokyo, Tōkyō shoseki 東京書籍, $112 \mathrm{p}$.

SHIGEMATSU Kiyoshi 重松清 2015 Tanpopo danchi たんぽぽ団地 (Le grand ensemble aux pissenlits), Tokyo, Shinchōsha 新潮社, $364 \mathrm{p}$.

SHIKI Koichi 志岐祐一 (éd.) 2012 Sekai ichi utsukushi danchi zukan 世界一 美しい団地図鑑 (Encyclopédie illustrée 
des plus beaux grands ensembles du monde), Tokyo, Ekusunaredji エクスナレ ッジ, 135 p.

Tōkyōjin 371 gō tokushū «Tōkyō natsukashi no danchi » 東京人371号特集 「東京なつかしの団地」(Tōkyōjin n³ 371, édition spéciale «Les grands ensembles tokyoïtes de nos souvenirs») 2016, Tokyo, Toshi shuppan 都市出版, 146 p.

\section{Autres références}

«Ryōsan sareru "genkai danchi"» 量産さ れる「限界団地」(Production de masse des danchis seuils), Asahi Shinbun 朝日新聞, 5 janvier 2009, p. 29.

\section{BESTOR Theodore C. 1989}

Neighborhood Tokyo, Stanford, CA, Stanford University Press, xvi-347 p.

\section{LE BRAS Hervé \& CHESNAIS Jean-} Claude 1976

"Cycle de l'habitat et âge des habitants», Population, vol. 31 (2) : 269-299.

\section{HARADA Sumitaka 原田純孝 1985} "Sengo jūtaku hōsei no seiritsu katei » 戦後住宅法制の成立過程 (Le processus de formation de la législation sur le logement dans l'après-guerre), Fukushi $k o k k a$ 福祉国家, 6 : 317-396.

\section{HIRAYAMA Yosuke 2003}

"Home-ownership in an unstable world: the case of Japan », in Forrest Ray \& Lee James (dir.), Housing and Social Change: East-West Perspectives, «Housing and
Society Series », Londres; New York, Routledge : 140-161.

HIRAYAMA Yōsuke 平山洋介 2006 Tōkyō no hate ni 東京の果てに (Aux confins de Tokyo), Tokyo, NTT shuppan NTT出版, 298 p.

HIRAYAMA Yōsuke 平山洋介 2009 Jūtaku seisaku no doko ga mondai ka : «mochi ie shakai» no tsugi o tenbō suru 住宅政策のどこが問題か:〈持家社会〉の次を 展望する (Les problèmes de la politique de logement : envisager un après de la « société de propriétaires»), Tokyo, Kōbunsha 光文社, $310 p$.

Housing Policy Division, Housing Bureau, Ministry of Land, Infrastructure, Transport and Tourism, Urban Renaissance Agency, Japan Housing Finance Agency \& The Building Center of Japan (dir.) 2016 A Quick Look at Housing in Japan, Tokyo, The Building Center of Japan, 94 p.

INABA Nanako 稲葉奈々子 2015 "Tōkyō orinpikku to toei kasumigaoka apāto» 東京オリンピックと都営霞が丘ア パート (Les Jeux olympiques de Tokyo et les appartements municipaux de Kasumigaoka), Yoseba 寄せ場, 27 :61-75.

Keizai kikaku chō 経済企画庁 1961 Kokumin seikatsu hakusho shōwa 35 nen ban 国民生活白書 昭和35年版 (Le livre blanc de la vie des citoyens, édition 1960), Tokyo, Keizai kikaku chō 経済企画庁, 231 p. 
Kōei jūtaku seido kenkyū kai 公営住宅 制度研究会 (dir.) 2006

Chiiki jūtaku tokubetsu sochi hō kaisei kōei jūtaku hō tō no kaisetsu 地域住宅 特別措置法·改正公営住宅法等の解説 (Commentaire de la loi sur les mesures territoriales spéciales sur le logement, les révisions de la loi sur le logement public et autres), Tokyo, Gyōsei ぎょうせい, 197 p.

Kokudo kōtsu shō 国土交通省 2009 Heisei 20 nendo kokudo kōtsū hakusho 平成20年度国土交通白書 (Livre blanc 2008 du ministère de l'aménagement du Territoire, des Infrastructures, des Transports et du Tourisme), Tokyo, Kokudo kōtsu shō 国土交通省, 225 p.

\section{LAÉ Jean-François \& MURARD Numa} 1985

L'argent des pauvres: la vie quotidienne en cité de transit, Paris, Seuil, 209 p.

\section{MARSHALL Thomas Humphrey \&} BOTTOMORE Tom [1950] 1992

Citizenship and Social Class, Londres \& Ann Arbor, Ml, Pluto Press, 102 p.

Meguro Kuyakusho 目黒区役所 2016 «Toei jūtaku no mōshikomi » 都営住宅 の申し込み (Comment demander un logement public), Meguro-ku 目黒区, 1er décembre, consulté le 6 juin 2017. https://www.city.meguro.tokyo.jp/kurashi/ sumai/moshikomi/toeijyutaku-setsumei. html

MORI Chikako 2013

«Esquisse d'une sociologie des banlieues au Japon ", Hommes \& Migrations, 1302 : 45-55.

Naikaku fu 内閣府 2016

Heisei 28 nen ban kōrei shakai hakusho 平成28年版高齢社会白書 (Livre blanc du vieillissement de la société, édition 2016) : 17-180.

NAKAZAWA Takumi 中沢卓実 \& Shukutoku daigaku kodokushi kenkyūkai 淑徳大学孤独死研究会 (dir.) 2008

Danchi to kodokushi 団地と孤独死 (Grands ensembles et morts solitaires), Tokyo, Chūōhōkishuppan 中央法規出版， $158 \mathrm{p}$.

\section{NEITZEL Laura 2016}

The Life We Longed for: Danchi Housing and the Middle Class Dream in Postwar Japan, Portland, ME, MerwinAsia, xxvi-159 p.

ŌMOTO Keino 大本圭野 (dir.) 1991 «Shōgen» nihon no jūtaku seisaku「証 言」日本の住宅政策 (Témoignages : la politique de logement japonaise), Tokyo, Nihon hyōronsha 日本評論社, xvii-907 p.

ŌNO Akira 大野晃 2005

Sanson kankyō shakaigaku shosetsu : gendai sanson no genkai shūrakuka to ryūiki kyōdō kanri 山村環境社会学 序説: 現代山村の限界集落化と流域共同 管理 (Introduction à la sociologie des villages de montagne : la transformation actuelle des villages de montagnes en villages seuils et la gestion collective des vallées), Tokyo, Nōsangyoson bunka kyōkai 農山漁村文化協会, 298 p. 
ŌNO Akira 大野晃 2008

Genkai shūraku to chiiki saisei 限界集

落と地域再生 (Les villages seuils et la régénération des territoires), Kōchi, Kōchi shinbunsha 高知新聞社, $313 \mathrm{p}$.

\section{P. N. 1960}

"Villes nouvelles en Angleterre», Population, vol. 15 (1) : 135-141.

\section{POUCHELLE Marie-Christine 1974}

Vivre dans un grand ensemble: " Les cimentiers», Paris, Épi, «Passé et présent», 1974, $110 \mathrm{p}$.

\section{RONALD Richard 2004}

«Home Ownership, Ideology and Diversity: Re-evaluating Concepts of Housing Ideology in the Case of Japan », Housing, Theory and Society, vol. 21 (2) : 49-64.

\section{SATŌ Iwao 佐藤岩夫 1999}

Gendai kokka to ippan jōkō : shakkahō no hikaku rekishi shakaikagakuteki kenkyū 現代国家と一般条項: 借家法の比較歴 史社会学的研究 (L'état moderne et les clauses générales : sociologie historique comparative des lois sur les logements en location), Tokyo, Sōbunsha 創文社, $338 \mathrm{p}$.

\section{SATŌ Iwao 2007}

«Welfare Regime Theories and the Japanese Housing System », in Hirayama Yōsuke \& Ronald Richard (dir.), Housing and Social Transition in Japan, «Housing and Society Series ", Abingdon; New York, NY, Routledge : 73-93.
SCHWARTZ Olivier [1990] 2009

Le monde privé des ouvriers : hommes et femmes du Nord, $2^{2}$ éd., Paris, PUF, I-531 p.

SERA Tomoki 瀬良智機 2015

UR Toshi kikō ni okeru jūtaku sēfuti netto e no torikumi UR都市機構における住宅セ一フ ティネットへの取組 (Les engagements de I'Agence urbaine UR en faveur d'un filet de sécurité du logement), 8 septembre, consulté le 2 juin 2017.

https://www.mlit.go.jp/ common/001102728.pdf

Sōmushō tōkei kyoku 総務省統計局 2010

Kokusei chōsa heisei nijūni nen 国勢調査 平成22年 (Recensement de 2010).

SUMIMOTO Yasushi 住本靖, IURA Yoshinori 井浦義典, KITA Katsuhiko 喜 多功彦 \& MATSUHIRA Kensuke 松平健輔 [2008] 2012

Chikujō kaisetsu kōei jūtaku hō 逐条解 説公営住宅法 (La Loi sur le logement public expliquée article par article), édition révisée, Tokyo, Gyōsei ぎょうせい, $\mathrm{xvi}-221 \mathrm{p}$.

Tōkyōto jūtaku kyōkyū kōsha 東京都住 宅供給公社 2017 «Shotoku kijun hyō (tanshinsha kazoku)» 所得基準表 (単身者·家族) (Tableau des critères de revenus (célibataires, familles)), consulté le 2 juin 2017, à l'adresse http://www.to-kousya. or.jp/opencms/export/sites/default/ www_to-kousya_or_jp/toeibosyu/sikaku/ shotoku_kijyun.pdf 
Tōkyōto jūtaku kyōkyū sōmu bu jūtaku seisaki shitsu - kaihatsu chōsei bu jūtaku keikaku ka 東京都住宅供給総務部 住宅政策室·開発調整部住宅計画課 (dir.) 2002

Tōkyōto jūtaku masutā puran: yutaka de iki iki to shita tōkyō kyojū no jitsugen o mezashite: 2001-2005 東京都住宅マスター プラン: 豊かでいきいきとした東京居住の実 現をめざして: 2001-2015 (Grand plan de logement du gouvernement de Tokyo : pour créer des résidences confortables et dynamiques à Tokyo), Tokyo, Tōkyōto 東京都, 136-26 p.

Tōkyōto jūtaku kyōkyū sōmu bu kikaku shitsu - kaihatsu chōsei bu tomin jūtaku shitsu 東京都住宅供給総務部企画 室·開発調整部都民住宅室 (dir.) 1991 Tōkyōto jūtaku masutā puran: yutori aru jūseikatsu no jitsugen o mezashite 東京 都住宅マスタープラン: ゆとりある住生活 の実現をめざして (Plan directeur pour le logement du gouvernement de Tokyo : pour créer de bonnes conditions d'habitat), Tokyo, Tōkyōto 東京都, 150 p.

TSUJINAKA Yutaka, PEKKANEN Robert \& YAMAMOTO Hidehiro 2014

Neighborhood Associations and Local Governance in Japan, Londres; New York, Routledge, xxiv-255 p.
Toshi saisei kikō UR都市再生機構 2017 «Omōshikomi shikaku ni tsuite (senchakujun)»お申込み資格について( 先着順) (Conditions requises pour faire une demande de logement (principe du premier arrivé, premier servi)), UR Toshi saisei kikō UR都市再生機構, consulté le 2 juin 2017.

http://www.ur-net.go.jp/tebiki/a-license. html

WATANABE Noriko 渡辺紀子 2015

«Toei jūtaku no ima : mondai to undō » 都営住宅の今: 問題と運動 (Les logements publics municipaux d'aujourd'hui : problèmes et mobilisations), Kyojū fukushi kenkyū 居住福祉研究, 19: 29-35. 